\title{
Some existence and uniqueness theorems on ordered metric spaces via generalized distances
}

\author{
Fayyaz Rouzkard ${ }^{1}$, Mohammad Imdad ${ }^{1}$ and Dhananjay Gopal ${ }^{*}$
}

"Correspondence:

gopal.dhananjay@rediffmail.com;

gopaldhananjay@yahoo.in

${ }^{2}$ Department of Applied

Mathematics \& Humanities, S. V.

National Institute of Technology,

Surat, 395007, India

Full list of author information is

available at the end of the article

\begin{abstract}
The purpose of this paper is to prove some fixed point theorems in a complete metric space equipped with a partial ordering using $w$-distances together with the aid of altering functions.
\end{abstract}

MSC: $54 \mathrm{H} 25 ; 47 \mathrm{H} 10$

Keywords: partially ordered set; fixed point; complete metric space; altering functions; $w$-distance; nondecreasing; orbitally continuous; orbitally $\mathcal{U}$-continuous

\section{Introduction with preliminaries}

The concept of a $w$-distance was initiated by Kada, Suzuki and Takahashi [1] and was primarily utilized to improve Caristi's fixed point theorem [2], Ekeland's variational principle [3] and nonconvex minimization theorems whose descriptions and details are available in Takahashi [4]. Proving existence results of fixed points on partially ordered metric spaces has been a relatively new development in metric fixed points theory. In [5], an analogue of Banach's fixed point theorem in a partially ordered metric space has been proved besides discussing some applications to matrix equations. Ran and Reurings have further weakened the usual contraction condition but merely up to monotone operators.

Branciari [6] established a fixed point result for an integral-type inequality, which is a generalization of the Banach contraction principle. Vijayaraju et al. [7] obtained a general principle, which made it possible to prove many fixed point theorems for pairs of maps satisfying integral-type contraction conditions.

Several fixed point and common fixed point theorems in metric and semi-metric spaces for compatible, weakly compatible and owc mappings satisfying contractive conditions of integral type were proved in [7-9] and in other papers. Later on, Suzuki [10] proved that integral-type contractions are Meir-Keeler contractions. He also showed that MeirKeeler contractions of integral type are still Meir-Keeler contractions. Jachymski [11] also proved that most contractive conditions of integral type given recently by many authors coincide with classical ones. But he gave a new contractive condition of integral type which is independent of classical ones. Recently Popa and Mocanu [9] obtained integral-type contractions via an altering distance function and proved general common fixed point results for integral-type contractive conditions.

In [12], Razani et al. proved a fixed point theorem for $(\phi, \psi, p)$-contractive mappings on $\mathcal{X}$ [i.e., for each $x, y \in \mathcal{X}, \phi p(\mathcal{T} x, \mathcal{T} y) \leq \psi \phi p(x, y)$ ], which is a new version of the main 
theorem in [6], by considering the concept of a $w$-distance. In fact, he proved the following result.

Theorem [12] Let p be a $w$-distance on a complete metric space $(\mathcal{X}, d), \phi$ be nondecreasing, continuous and $\phi(\epsilon)>0$ for each $\epsilon>0$ and $\psi$ be nondecreasing, right continuous and $\psi(t)<$ $t$ for all $t>0$. Suppose $\mathcal{T}$ is a $(\phi, \psi, p)$-contractive map on $\mathcal{X}$, then $\mathcal{T}$ has a unique fixed point in $\mathcal{X}$. Moreover, $\lim _{n \rightarrow \infty} \mathcal{T}^{n} x$ is a fixed point of $\mathcal{T}$ for each $x \in \mathcal{X}$.

In [13] Lakzian and Lin obtained some generalizations of fixed point theorems by Kada et al. [1], Hicks and Rhoades [14] and several other results with respect to $(\phi, \psi, p)$ contractive maps on a complete metric space.

In this paper, we use the concept of a $w$-distance to prove the fixed point theorems in partially ordered metric spaces. Our results do not only generalize some fixed point theorems, but also improve and simplify the previous results.

Before presenting our results, we collect relevant definitions and results which will be needed in our subsequent discussion.

Definition 1 Let $\mathcal{X}$ be a nonempty set. Then $(\mathcal{X}, d, \preceq)$ is called a partially ordered metric space if

(i) $(\mathcal{X}, \preceq)$ is a partially ordered set and

(ii) $(\mathcal{X}, d)$ is a metric space.

Definition 2 Let $(\mathcal{X}, \preceq)$ be a partially ordered set. Then

(a) elements $x, y \in \mathcal{X}$ are called comparable with respect to ' $\preceq$ ' if either $x \preceq y$ or $y \preceq x$;

(b) a mapping $\mathcal{T}: \mathcal{X} \rightarrow \mathcal{X}$ is called nondecreasing with respect to ' $\preceq$ ' if $x \preceq y$ implies $\mathcal{T} x \preceq \mathcal{T} y$.

Definition 3 [1] Let $(\mathcal{X}, d)$ be a metric space. Then the function $p: \mathcal{X} \times \mathcal{X} \rightarrow[0, \infty)$ is called a $w$-distance on $\mathcal{X}$ if the following conditions are satisfied:

(a) $p(x, z) \leq p(x, y)+p(y, z)$ for any $x, y, z \in \mathcal{X}$,

(b) for any $x \in \mathcal{X}, p(x, \cdot): \mathcal{X} \rightarrow[0, \infty)$ is lower semi-continuous, i.e., if $x \in \mathcal{X}$ and $y_{n} \rightarrow y$ in $\mathcal{X}$, then $p(x, y) \leq \liminf _{n} p\left(x, y_{n}\right)$,

(c) for any $\epsilon>0$, there exists $\delta>0$ such that $p(x, z) \leq \delta$ and $p(z, y) \leq \delta$ imply $d(x, y) \leq \epsilon$.

Example 1 [15] Let $(\mathcal{X}, d)$ be a metric space and let $g$ be a continuous mapping from $\mathcal{X}$ into itself. Then a function $p: \mathcal{X} \times \mathcal{X} \rightarrow[0, \infty)$ defined by

$$
p(x, y)=\max \{d(g x, y), d(g x, g y)\} \quad \text { for every } x, y \in \mathcal{X}
$$

is a $w$-distance on $\mathcal{X}$.

Clearly, every metric is a $w$-distance but not conversely. The following example substantiates this fact.

Example 2 Let $(\mathcal{X}, d)$ be a metric space. A function $p: \mathcal{X} \times \mathcal{X} \rightarrow[0, \infty)$ defined by $p(x, y)=k$ for every $x, y \in \mathcal{X}$ is a $w$-distance on $\mathcal{X}$, where $k$ is a positive real number. But $p$ is not a metric since $p(x, x)=k \neq 0$ for any $x \in \mathcal{X}$. 
Example 3 Let $\mathcal{X}=\{1,2,3,4\}$ be a metric space with a metric

$$
d(x, y)=|x-y| \quad \text { for all } x, y \in \mathcal{X} .
$$

Let $p: \mathcal{X} \times \mathcal{X} \rightarrow[0, \infty)$ be defined by

$$
\begin{aligned}
& p(1,2)=p(2,1)=3, \quad p(1,3)=p(3,1)=p(2,3)=p(3,2)=1, \\
& p(1,4)=p(4,1)=p(2,4)=p(4,2)=p(3,4)=p(4,3)=2,
\end{aligned}
$$

and $p(x, x)=0.6$ for every $x \in \mathcal{X}$.

Clearly, conditions (b) and (c) from the definition of $w$-distance are satisfied (for every $\epsilon>0$, put $\delta=\frac{1}{2}$ ), while condition (a) is not satisfied as

$$
p(1,2)=3>1+1=p(1,3)+p(3,2) .
$$

Definition 4 Let $\mathcal{T}: \mathcal{X} \rightarrow \mathcal{X}$ be a function.

(a) $\mathcal{F}_{\mathcal{T}}=\{x \in \mathcal{X} \mid x=\mathcal{T}(x)\}$ (i.e., a set of fixed points of $\mathcal{T}$ ).

(b) The function $\mathcal{T}$ is called a Picard operator (briefly, PO) if there exists $x^{*} \in \mathcal{X}$ such that $\mathcal{F}_{\mathcal{T}}=\left\{x^{*}\right\}$ and $\left\{\mathcal{T}^{n}(x)\right\}$ converges to $x^{*}$ for all $x \in \mathcal{X}$.

(c) The function $\mathcal{T}$ is called orbitally $\mathcal{U}$-continuous for any $\mathcal{U} \subset \mathcal{X} \times \mathcal{X}$ if the following condition is satisfied:

For any $x \in \mathcal{X}, \mathcal{T}^{n_{i}}(x) \rightarrow a \in \mathcal{X}$ as $i \rightarrow \infty$ and $\left(\mathcal{T}^{n_{i}}(x), a\right) \in \mathcal{U}$ for any $i \in N$ imply that $\mathcal{T}^{n_{i}+1}(x) \rightarrow \mathcal{T} a$ as $i \rightarrow \infty$.

Let $(\mathcal{X}, \preceq)$ be a partially ordered set. Let us denote by $\mathcal{X}_{\leq}$the subset of $\mathcal{X} \times \mathcal{X}$ defined by

$$
\mathcal{X}_{\leq}=\{(x, y) \in \mathcal{X} \times \mathcal{X} \mid x \preceq y \text { or } y \preceq x\} .
$$

Definition 5 A map $\mathcal{T}: \mathcal{X} \rightarrow \mathcal{X}$ is said to be orbitally continuous if $x \in \mathcal{X}$ and $\mathcal{T}^{n_{i}}(x) \rightarrow$ $a \in \mathcal{X}$ as $i \rightarrow \infty$ implies that $\mathcal{T}^{n_{i}+1}(x) \rightarrow \mathcal{T} a$ as $i \rightarrow \infty$.

\section{Suppose}

$$
\begin{aligned}
\Phi= & \{\phi \mid \phi:[0, \infty) \rightarrow[0, \infty) \text { is nondecreasing, continuous and } \\
& \phi(\epsilon)>0 \text { for each } \epsilon>0\} .
\end{aligned}
$$

Moreover, let

$$
\begin{aligned}
\Psi= & \{\psi \mid \psi:[0, \infty) \rightarrow[0, \infty) \text { is nondecreasing, right continuous and } \\
& \psi(t)<t \text { for all } t>0\} .
\end{aligned}
$$

Also, let

$$
\begin{aligned}
\Gamma= & \{\gamma \mid \gamma:[0, \infty) \rightarrow[0, \infty) \text { is nondecreasing, continuous and } \\
& \gamma(t)=0 \text { iff } t=0\} \quad(c f .[16]) .
\end{aligned}
$$


Example 4 Let $\left\{a_{n}\right\}_{n=1}^{\infty}$ and $\left\{c_{n}\right\}_{n=0}^{\infty}$ be two non-negative sequences such that $\left\{a_{n}\right\}$ is strictly decreasing and converging to zero, and (for each $n \in \mathbb{N}) c_{n-1} a_{n}>a_{n+1}$, where $0<c_{n-1}<1$. Define $\psi:[0, \infty) \rightarrow[0, \infty)$ by $\psi(0)=0, \psi(t)=c_{n} t$, if $a_{n+1} \leq t<a_{n}, \psi(t)=c_{0} t$ if $t \geq a_{1}$, then $\psi$ is in $\Psi$.

Now, we prove the following two lemmas.

Lemma 1 If $\psi \in \Psi$, then $\lim _{n \rightarrow \infty} \psi^{n}(t)=0$ for each $t>0$.

Proof Owing to the monotonicity of $\psi$, for each $t>0,\left\{\psi^{n}(t)\right\}$ is non-increasing and also non-negative. Thus, there exists $\alpha \geq 0$ such that $\alpha^{+}=\lim _{n \rightarrow \infty} \psi^{n}(t)$. Suppose on the contrary that $\alpha>0$. As $\psi$ is right continuous, therefore

$$
0<\alpha=\lim _{n \rightarrow \infty} \psi^{n+1}(t)=\psi(\alpha)
$$

which is a contradiction as $\psi(t)<t$. Thus $\alpha=0$.

Lemma 2 If $\phi \in \Phi(\gamma \in \Gamma),\left\{a_{n}\right\} \subset[0, \infty)$ and $\lim _{n \rightarrow \infty} \phi\left(a_{n}\right)=0\left(\lim _{n \rightarrow \infty} \gamma\left(a_{n}\right)=0\right)$, then $\lim _{n \rightarrow \infty} a_{n}=0$.

Proof If there exists $\epsilon>0$ and $\left\{n_{k}\right\}_{k=1}^{\infty}$ such that

$$
a_{n_{k}} \geq \epsilon>0
$$

then

$$
\limsup _{n \rightarrow \infty} \phi\left(a_{n}\right) \geq \limsup _{k \rightarrow \infty} \phi\left(a_{n_{k}}\right) \geq \phi(\epsilon)>0
$$

yielding thereby $\lim _{n \rightarrow \infty} \phi\left(a_{n}\right) \neq 0$.

The following two lemmas are crucial in the proofs of our main results.

Lemma $3[1,17]$ Let $(\mathcal{X}, d)$ be a metric space equipped with a $w$-distance $p$. Let $\left\{x_{n}\right\}$ and $\left\{y_{n}\right\}$ be sequences in $\mathcal{X}$ whereas $\left\{\alpha_{n}\right\}$ and $\left\{\beta_{n}\right\}$ be sequences in $[0, \infty)$ converging to zero. Then the following conditions hold (for $x, y, z \in \mathcal{X}$ ):

(a) if $p\left(x_{n}, y\right) \leq \alpha_{n}$ and $p\left(x_{n}, z\right) \leq \beta_{n}$ for $n \in N$, then $y=z$. In particular, if $p(x, y)=0$ and $p(x, z)=0$, then $y=z$

(b) if $p\left(x_{n}, y_{n}\right) \leq \alpha_{n}$ and $p\left(x_{n}, z\right) \leq \beta_{n}$ for $n \in N$, then $\lim _{n \rightarrow \infty} d\left(y_{n}, z\right)=0$;

(c) if $p\left(x_{n}, x_{m}\right) \leq \alpha_{n}$ for $n, m \in N$ with $m>n$, then $\left\{x_{n}\right\}$ is a Cauchy sequence;

(d) if $p\left(y, x_{n}\right) \leq \alpha_{n}$ for $n \in N$, then $\left\{x_{n}\right\}$ is a Cauchy sequence.

Lemma 4 [1] Let $p$ be a $w$-distance on a metric space $(\mathcal{X}, d)$ and $\left\{x_{n}\right\}$ be a sequence in $\mathcal{X}$ such that for each $\epsilon>0$, there exists $N_{\epsilon} \in N$ such that $m>n>N_{\epsilon}$ implies $p\left(x_{n}, x_{m}\right)<\epsilon$ (or $\left.\lim _{m, n} p\left(x_{n}, x_{m}\right)=0\right)$. Then $\left\{x_{n}\right\}$ is a Cauchy sequence. 


\section{$2(\phi, \psi, p)$-contractive maps}

Now, we present our main result as follows.

Theorem 1 Let $(\mathcal{X}, d, \preceq)$ be a complete partially ordered metric space equipped with a $w$-distance $p$ and $\mathcal{S}: \mathcal{X} \rightarrow \mathcal{X}$ be nondecreasing mapping. Suppose that

(a) there exists $x_{0} \in \mathcal{X}$ such that $\left(x_{0}, \mathcal{S} x_{0}\right) \in \mathcal{X}_{\leq}$,

(b) there exist $\psi \in \Psi$ and $\phi \in \Phi$ such that

$$
\phi(p(\mathcal{S} x, \mathcal{S} y)) \leq \psi \phi(p(x, y))
$$

for all $(x, y) \in \mathcal{X}_{\leq}$

(c) either $\mathcal{S}$ is orbitally continuous at $x_{0}$ or

$\left(c^{\prime}\right) \mathcal{S}$ is orbitally $\mathcal{X}_{\leq}$-continuous and there exists a subsequence $\left\{\mathcal{S}^{n_{k}} x_{0}\right\}$ of $\left\{\mathcal{S}^{n} x_{0}\right\}$ converging to $x^{*}$ such that $\left(\mathcal{S}^{n_{k}} x_{0}, x^{*}\right) \in \mathcal{X}_{\leq}$for any $k \in N$.

Then $\mathcal{F}_{\mathcal{S}} \neq \emptyset$. Moreover, if $x=\mathcal{S} x$, then $p(x, x)=0$.

Proof If $x_{0}=\mathcal{S} x_{0}$ for some $x_{0} \in \mathcal{X}$, then there is nothing to prove. Otherwise, let there be $x_{0} \in \mathcal{X}$ such that $x_{0} \neq \mathcal{S} x_{0}$, and $\left(x_{0}, \mathcal{S} x_{0}\right) \in \mathcal{X}_{\preceq}$. Owing to the monotonicity of $\mathcal{S}$, we can write $\left(\mathcal{S} x_{0}, \mathcal{S}^{2} x_{0}\right) \in \mathcal{X}_{\leq}$. Continuing this process inductively, we obtain

$$
\left(\mathcal{S}^{n} x_{0}, \mathcal{S}^{m} x_{0}\right) \in \mathcal{X}_{\preceq}
$$

for any $n, m \in N$. Now, we proceed to show that

$$
\lim _{n \rightarrow \infty} p\left(\mathcal{S}^{n} x_{0}, \mathcal{S}^{n+1} x_{0}\right)=0 .
$$

By using condition (b) and the properties of $\phi$, $\psi$, we get

$$
\begin{aligned}
\phi\left(p\left(\mathcal{S}^{n} x_{0}, \mathcal{S}^{n+1} x_{0}\right)\right) & \leq \psi \phi\left(p\left(\mathcal{S}^{n-1} x_{0}, \mathcal{S}^{n} x_{0}\right)\right) \\
& \leq \psi^{2} \phi\left(p\left(\mathcal{S}^{n-2} x_{0}, \mathcal{S}^{n-1} x_{0}\right)\right) \\
& \leq \cdots \\
& \leq \psi^{n-m} \phi\left(p\left(\mathcal{S}^{m} x_{0}, \mathcal{S}^{m+1} x_{0}\right)\right) \\
& \leq \cdots \\
& \leq \psi^{n} \phi\left(p\left(x_{0}, \mathcal{S} x_{0}\right)\right) .
\end{aligned}
$$

Now, using Lemma $1, \lim _{n \rightarrow \infty} \phi\left(p\left(\mathcal{S}^{n} x_{0}, \mathcal{S}^{n+1} x_{0}\right)\right)=0$, which due to Lemma 2 gives rise to

$$
\lim _{n \rightarrow \infty} p\left(\mathcal{S}^{n} x_{0}, \mathcal{S}^{n+1} x_{0}\right)=0
$$

so that (1) is established.

Similarly, we can show

$$
\lim _{n \rightarrow \infty} p\left(\mathcal{S}^{n+1} x_{0}, \mathcal{S}^{n} x_{0}\right)=0
$$


Next, we proceed to show

$$
\lim _{n, m \rightarrow \infty} p\left(\mathcal{S}^{n} x_{0}, \mathcal{S}^{m} x_{0}\right)=0
$$

Suppose (4) is untrue. Then we can find a $\delta>0$ with sequences $\left\{m_{k}\right\}_{k=1}^{\infty},\left\{n_{k}\right\}_{k=1}^{\infty}$ such that

$$
p\left(\mathcal{S}^{n_{k}} x_{0}, \mathcal{S}^{m_{k}} x_{0}\right) \geq \delta, \quad \text { for all } k \in\{1,2,3, \ldots\}
$$

wherein $m_{k}>n_{k}$. By (1) there exists $k_{0} \in \mathbb{N}$ such that $n_{k}>k_{0}$ implies

$$
p\left(\mathcal{S}^{n_{k}} x_{0}, \mathcal{S}^{n_{k}+1} x_{0}\right)<\delta
$$

Notice that in view of (5) and (6), $m_{k} \neq n_{k+1}$. We can assume that $m_{k}$ is a minimum index such that (5) holds so that

$$
p\left(\mathcal{S}^{n_{k}} x_{0}, \mathcal{S}^{r} x_{0}\right)<\delta, \quad \text { for } r \in\left\{n_{k+1}, n_{k+2}, \ldots, m_{k}-1\right\} .
$$

Now (1), (5) and (7) imply

$$
\begin{aligned}
o & <\delta \leq p\left(\mathcal{S}^{n_{k}} x_{0}, \mathcal{S}^{m_{k}} x_{0}\right) \\
& \leq p\left(\mathcal{S}^{n_{k}} x_{0}, \mathcal{S}^{m_{k}-1} x_{0}\right)+p\left(\mathcal{S}^{m_{k}-1} x_{0}, \mathcal{S}^{m_{k}} x_{0}\right) \\
& <\delta+p\left(\mathcal{S}^{m_{k}-1} x_{0}, \mathcal{S}^{m_{k}} x_{0}\right)
\end{aligned}
$$

so that

$$
\lim _{k \rightarrow \infty} p\left(\mathcal{S}^{n_{k}} x_{0}, \mathcal{S}^{m_{k}} x_{0}\right)=\delta
$$

If $\epsilon=\lim \sup _{k} p\left(\mathcal{S}^{n_{k}+1} x_{0}, \mathcal{S}^{m_{k}+1} x_{0}\right) \geq \delta$, then there exists $\left\{k_{r}\right\}_{r=1}^{\infty}$ such that

$$
\lim _{r \rightarrow \infty} p\left(\mathcal{S}^{n_{k_{r}}+1} x_{0}, \mathcal{S}^{m_{k_{r}}+1} x_{0}\right)=\epsilon \geq \delta .
$$

Since $\phi$ is continuous and nondecreasing and also $\left(\mathcal{S}^{n_{k_{r}}} x_{0}, \mathcal{S}^{m_{k_{r}}} x_{0}\right) \in \mathcal{X}_{\leq}$, by using condition (b) and (8), one gets

$$
\phi(\delta) \leq \phi(\epsilon)=\lim _{r \rightarrow \infty} \phi\left(p\left(\mathcal{S}^{n_{k_{r}}+1} x_{0}, \mathcal{S}^{m_{k_{r}}+1} x_{0}\right)\right) \leq \psi \phi(\delta) .
$$

Notice that

$$
\phi p\left(\mathcal{S}^{n_{k_{r}}} x_{0}, \mathcal{S}^{m_{k_{r}}} x_{0}\right) \rightarrow \phi(\delta)^{+}
$$

and $\psi$ is right continuous, therefore $\phi(\delta)=0$. This is indeed a contradiction and

$$
\limsup _{k} p\left(\mathcal{S}^{n_{k}+1} x_{0}, \mathcal{S}^{m_{k}+1} x_{0}\right)<\delta
$$


so we have

$$
\begin{aligned}
\delta & \leq p\left(\mathcal{S}^{n_{k}} x_{0}, \mathcal{S}^{m_{k}} x_{0}\right) \\
& \leq p\left(\mathcal{S}^{n_{k}} x_{0}, \mathcal{S}^{n_{k}+1} x_{0}\right)+p\left(\mathcal{S}^{n_{k}+1} x_{0}, \mathcal{S}^{m_{k}+1} x_{0}\right)+p\left(\mathcal{S}^{m_{k}+1} x_{0}, \mathcal{S}^{m_{k}} x_{0}\right) \\
& \leq \lim _{k \rightarrow \infty} p\left(\mathcal{S}^{n_{k}} x_{0}, \mathcal{S}^{n_{k}+1} x_{0}\right)+\limsup _{k} p\left(\mathcal{S}^{n_{k}+1} x_{0}, \mathcal{S}^{m_{k}+1} x_{0}\right)+\lim _{k \rightarrow \infty} p\left(\mathcal{S}^{m_{k}+1} x_{0}, \mathcal{S}^{m_{k}} x_{0}\right) \\
& \left.=\limsup _{k} p\left(\mathcal{S}^{n_{k}+1} x_{0}, \mathcal{S}^{m_{k}+1} x_{0}\right)<\delta \quad \text { (on using }(1)\right),
\end{aligned}
$$

which is a contradiction. Thus, (4) is proved.

Owing to Lemma $4,\left\{\mathcal{S}^{n} x_{0}\right\}$ is a Cauchy sequence in $\mathcal{X}$. Since $\mathcal{X}$ is a complete metric space, there exists $x^{*} \in \mathcal{X}$ such that $\mathcal{S}^{n} x_{0} \rightarrow x^{*}$ as $n \rightarrow \infty$.

Now, we show that $x^{*}$ is a fixed point of $\mathcal{S}$. If (c) holds, then $\mathcal{S}^{n+1} x_{0} \rightarrow \mathcal{S} x^{*}$ (as $\left.n \rightarrow \infty\right)$. By the lower semi-continuity of $p\left(\mathcal{S}^{n} x_{0}, \cdot\right)$, we have

$$
\begin{aligned}
& p\left(\mathcal{S}^{n} x_{0}, x^{*}\right) \leq \liminf _{m \rightarrow \infty} p\left(\mathcal{S}^{n} x_{0}, \mathcal{S}^{m} x_{0}\right)=\alpha_{n} \quad \text { (say), } \\
& p\left(\mathcal{S}^{n} x_{0}, \mathcal{S} x^{*}\right) \leq \liminf _{m \rightarrow \infty} p\left(\mathcal{S}^{n} x_{0}, \mathcal{S}^{m+1} x_{0}\right)=\beta_{n} \quad \text { (say). }
\end{aligned}
$$

By using (4), we have $\alpha_{n}, \beta_{n} \rightarrow 0$ as $n \rightarrow \infty$. Now, in view of Lemma 3, we conclude that

$$
\mathcal{S} x^{*}=x^{*}
$$

Next, suppose that $\left(\mathrm{c}^{\prime}\right)$ holds. Since $\left\{\mathcal{S}^{n_{k}} x_{0}\right\}$ converges to $x^{*},\left(\mathcal{S}^{n_{k}} x_{0}, x^{*}\right) \in \mathcal{X}_{\leq}$and $\mathcal{S}$ is $\mathcal{X}_{\leq}$-continuous, it follows that $\left\{\mathcal{S}^{n_{k}+1} x_{0}\right\}$ converges to $\mathcal{S} x^{*}$. As earlier, by the lower semicontinuity of $p\left(\mathcal{S}^{n} x_{0}, \cdot\right)$, we conclude that $\mathcal{S} x^{*}=x^{*}$.

If $\mathcal{S} x=x$, we have

$$
\phi p(x, x)=\phi p(\mathcal{S} x, \mathcal{S} x) \leq \psi \phi(p(x, x))<\phi p(x, x) .
$$

This is a contradiction which amounts to say that $\phi p(x, x)=0$ so that $p(x, x)=0$. This completes the proof.

The following example substantiates the fact that the condition of a partial ordering on the underlying metric space is necessary in Theorem 1.

Example 5 Consider $\mathcal{X}=[0,1]$ which is indeed a complete metric space under a usual metric $d(x, y)=|x-y|$ (for all $x, y \in \mathcal{X}$ ) wherein by defining $p(x, y)=3|x-y|$, we are in the receipt of a $w$-distance $p$ on $(\mathcal{X}, d)$. We consider $\mathcal{X}_{\preceq}$ as follows:

$$
\mathcal{X}_{\leq}=\left\{(x, y) \in \mathcal{X} \times \mathcal{X}: x=y \text { or } x, y \in\{0\} \cup\left\{\frac{1}{n}: n=1,2,3, \ldots\right\}\right\},
$$

where ' $\preceq$ ' is the usual ordering.

Let $\mathcal{S}: \mathcal{X} \rightarrow \mathcal{X}$ be given by

$$
\mathcal{S}(x)= \begin{cases}0, & \text { if } x=0 \\ \frac{1}{10 n-1}, & \text { if } x=\frac{1}{n} \\ \frac{1}{\sqrt{2}}, & \text { otherwise. }\end{cases}
$$


Obviously, $\mathcal{S}$ is a nondecreasing map. Also, there is $x_{0}=0$ in $\mathcal{X}$ such that $0=x_{0} \preceq \mathcal{S} x_{0}=0$, i.e., $\left(x_{0}, \mathcal{S} x_{0}\right) \in \mathcal{X}_{\leq}$, and $\mathcal{S}$ satisfies condition $\left(\mathrm{c}^{\prime}\right)$. We now show that $\mathcal{S}$ satisfies (b) wherein $\phi, \psi:[0, \infty) \rightarrow[0, \infty)$ are defined as

$$
\phi(t)=t^{\frac{1}{2}} \quad \text { and } \quad \psi(t)=\frac{1}{2} t(t \in[0, \infty)) .
$$

Clearly, $\phi \in \Phi$ and $\psi \in \Psi$. If $x=y$, condition (b) is satisfied.

Let $x=0$ and $y=\frac{1}{n}$, then

$$
\begin{aligned}
\phi p(\mathcal{S} x, \mathcal{S} y) & =\phi\left(\frac{3}{10 n-1}\right)=\left(\frac{3}{10 n-1}\right)^{\frac{1}{2}} \leq \frac{1}{2}\left(\frac{3}{n}\right)^{\frac{1}{2}} \\
& =\frac{1}{2} \phi(p(x, y))=\psi \phi(p(x, y)),
\end{aligned}
$$

as for any $n \in \mathbb{N},\left(\frac{n}{10 n-1}\right)^{\frac{1}{2}} \leq \frac{1}{2}$.

Next, let $x=\frac{1}{n}$ and $y=\frac{1}{m}$ with $m>n$. Then we have

$$
\begin{aligned}
\phi p(\mathcal{S} x, \mathcal{S} y) & =\phi\left(\frac{30(m-n)}{(10 n-1)(10 m-1)}\right) \\
& =\left(\frac{30(m-n)}{(10 n-1)(10 m-1)}\right)^{\frac{1}{2}} \leq \frac{1}{2}\left(\frac{3(m-n)}{m n}\right)^{\frac{1}{2}} \\
& =\frac{1}{2} \phi(p(x, y))=\psi \phi(p(x, y)),
\end{aligned}
$$

as for any $n \in \mathbb{N},\left(\frac{10 m n}{(10 n-1)(10 m-1)}\right)^{\frac{1}{2}} \leq \frac{1}{2}$. Hence condition (b) is satisfied.

Thus, all the conditions of Theorem 1 are satisfied implying thereby the existence of a fixed point of the map $\mathcal{S}$, which are indeed two in number, namely: $x=0, \frac{1}{\sqrt{2}}$.

Here, it can be pointed out that this example will not work in a metric space equipped with a $w$-distance without a partial ordering as condition (b) of Theorem 1 will not be satisfied. To substantiate this claim, choosing $x=\sqrt{2}$ and $y=0$ in condition (b), we get

$$
\phi p(\mathcal{S} \sqrt{2}, \mathcal{S} 0)=\left(\frac{3 \sqrt{2}}{2}\right)^{\frac{1}{2}} \not \notin \psi \phi(p(\sqrt{2}, 0))=\frac{(3 \sqrt{2})^{\frac{1}{2}}}{2},
$$

which is a contradiction.

In Theorem 1 , if $\mathcal{S}: \mathcal{X} \rightarrow \mathcal{X}$ is a continuous map, we deduce the following corollary.

Corollary 1 Let $(\mathcal{X}, d, \preceq)$ be a complete partially ordered metric space equipped with a $w$-distance $p$ and $\mathcal{S}: \mathcal{X} \rightarrow \mathcal{X}$ be a continuous and nondecreasing mapping. Suppose that

(a) there exists $x_{0} \in \mathcal{X}$ such that $\left(x_{0}, \mathcal{S} x_{0}\right) \in \mathcal{X}_{\leq}$,

(b) there exist $\phi \in \Phi$ and $\psi \in \Psi$ such that

$$
\phi p(\mathcal{S} x, \mathcal{S} y) \leq \psi \phi(p(x, y))
$$

for all $(x, y) \in \mathcal{X}_{\leq}$.

Then $\mathcal{F}_{\mathcal{S}} \neq \emptyset$. Moreover, if $x=\mathcal{S} x$, then $p(x, x)=0$. 
In Theorem 1, setting $\phi=\mathcal{I}$, the identity mapping, we deduce the following corollary.

Corollary 2 Let $(\mathcal{X}, d, \preceq)$ be a complete partially ordered metric space equipped with a $w$-distance $p$ and $\mathcal{S}: \mathcal{X} \rightarrow \mathcal{X}$ be a nondecreasing mapping. Suppose that

(a) there exists $x_{0} \in \mathcal{X}$ such that $\left(x_{0}, \mathcal{S} x_{0}\right) \in \mathcal{X}_{\leq}$,

(b) there exists $\phi \in \Psi$ such that

$$
p(\mathcal{S} x, \mathcal{S} y) \leq \psi(p(x, y))
$$

for all $(x, y) \in \mathcal{X}_{\leq}$,

(c) either $\mathcal{S}$ is orbitally continuous at $x_{0}$ or

$\left(c^{\prime}\right) \mathcal{S}$ is orbitally $\mathcal{X}_{\leq}$-continuous and there exists a subsequence $\left\{\mathcal{S}^{n_{k}} x_{0}\right\}$ of $\left\{\mathcal{S}^{n} x_{0}\right\}$ which converges to $x^{*}$ such that $\left(\mathcal{S}^{n_{k}} x_{0}, x^{*}\right) \in \mathcal{X}_{\leq}$for any $k \in N$.

Then $\mathcal{F}_{\mathcal{S}} \neq \emptyset$. Moreover, if $x=\mathcal{S} x$, then $p(x, x)=0$.

Choosing $\phi=\mathcal{I}$, the identity mapping and $\psi(t)=\alpha t$ (for all $t \in[0, \infty)$ and $\alpha \in[0,1)$ ) in Theorem 1, we deduce the following corollary.

Corollary 3 Let $(\mathcal{X}, d, \preceq)$ be a complete partially ordered metric space equipped with a $w$-distance $p$ and $\mathcal{S}: \mathcal{X} \rightarrow \mathcal{X}$ be a nondecreasing mapping. Suppose that

(a) there exists $x_{0} \in \mathcal{X}$ such that $\left(x_{0}, \mathcal{S} x_{0}\right) \in \mathcal{X}_{\leq}$,

(b) if

$$
p(\mathcal{S} x, \mathcal{S} y) \leq \alpha p(x, y)
$$

for all $(x, y) \in \mathcal{X}_{\leq}$, where $\alpha \in[0,1)$ and

(c) either $\mathcal{S}$ is orbitally continuous at $x_{0}$ or

$\left(c^{\prime}\right) \mathcal{S}$ is orbitally $\mathcal{X}_{\leq}$-continuous and there exists a subsequence $\left\{\mathcal{S}^{n_{k}} x_{0}\right\}$ of $\left\{\mathcal{S}^{n} x_{0}\right\}$ which converges to $x^{*}$ such that $\left(\mathcal{S}^{n_{k}} x_{0}, x^{*}\right) \in \mathcal{X}_{\leq}$for any $k \in N$.

Then $\mathcal{F}_{\mathcal{S}} \neq \emptyset$. Moreover, if $x=\mathcal{S} x$, then $p(x, x)=0$.

Suppose, $\omega: \mathbb{R}^{+} \rightarrow \mathbb{R}^{+}$is a Lebesgue-integrable mapping which is summable and $\int_{0}^{\epsilon} \omega(\xi) d \xi>0$ for each $\epsilon>0$. Now, in the next corollary, set $\phi(t)=\int_{0}^{t} \omega(\xi) d \xi$ and $\psi(t)=\alpha t$, where $\alpha \in[0,1)$ in Theorem 3. Clearly, $\phi \in \Phi$ and $\psi \in \Psi$. Hence, we can derive the following corollary as a special case.

Corollary 4 Let $(\mathcal{X}, d, \preceq)$ be a complete partially ordered metric space equipped with a $w$-distance $p$ and $\mathcal{S}: \mathcal{X} \rightarrow \mathcal{X}$ be a nondecreasing mapping. Suppose that

(a) there exists $x_{0} \in \mathcal{X}$ such that $\left(x_{0}, \mathcal{S} x_{0}\right) \in \mathcal{X}_{\leq}$,

(b) for all $(x, y) \in \mathcal{X}_{\leq}$,

$$
\int_{0}^{p(\mathcal{S} x, \mathcal{S} y)} \omega(\xi) d \xi \leq \alpha \int_{0}^{p(x, y)} \omega(\xi) d \xi
$$

(c) either $\mathcal{S}$ is orbitally continuous at $x_{0}$ or 
$\left(c^{\prime}\right) \mathcal{S}$ is orbitally $\mathcal{X}_{\leq}$-continuous and there exists a subsequence $\left\{\mathcal{S}^{n_{k}} x_{0}\right\}$ of $\left\{\mathcal{S}^{n} x_{0}\right\}$ which converges to $x^{*}$ such that $\left(\mathcal{S}^{n_{k}} x_{0}, x^{*}\right) \in \mathcal{X}_{\leq}$for any $k \in N$.

Then $\mathcal{F}_{\mathcal{S}} \neq \emptyset$. Moreover, if $x=\mathcal{S} x$, then $p(x, x)=0$.

The following simple example demonstrates Theorem 1.

Example 6 Consider $\mathcal{X}=\{(1,0),(0,1)\}$, which is a complete metric space with $d\left(\left(x_{1}, x_{2}\right)\right.$, $\left.\left(y_{1}, y_{2}\right)\right)=\left|x_{1}-y_{1}\right|+\left|x_{2}-y_{2}\right|$. Define $g: \mathcal{X} \rightarrow \mathcal{X}$ by $g(1,0)=(1,0)$ and $g(0,1)=(1,0)$, which is a continuous map. Moreover, by defining

$$
p\left(\left(x_{1}, x_{2}\right),\left(y_{1}, y_{2}\right)\right)=\max \left\{d\left(g\left(x_{1}, x_{2}\right),\left(y_{1}, y_{2}\right)\right), d\left(g\left(x_{1}, x_{2}\right), g\left(y_{1}, y_{2}\right)\right)\right\}
$$

$p$ is a $w$-distance on $(\mathcal{X}, d)$. For $(x, y)$ and $(z, t)$ in $\mathcal{X}$, define ' $\leq$ ' as follows: $(x, y) \preceq(z, t) \Leftrightarrow$ $x \leq z$ and $y \leq t$ (' $\leq$ ' is the usual ordering) so that

$$
\mathcal{X}_{\leq}=\{((1,0),(1,0)),((0,1),(0,1))\} .
$$

Let $\mathcal{S}: \mathcal{X} \rightarrow \mathcal{X}$ be given by

$$
\mathcal{S}(x, y)= \begin{cases}(1,0), & \text { if }(x, y)=(1,0), \\ (1,0), & \text { if }(x, y)=(0,1) .\end{cases}
$$

Clearly, $\mathcal{S}$ is a nondecreasing and continuous map. Also, $(1,0) \leq \mathcal{S}(1,0)=(1,0)$, i.e., $((1,0), \mathcal{S}(1,0)) \in \mathcal{X}_{\leq}$.

We now show that $\mathcal{S}$ satisfies (b) with $\phi, \psi:[0, \infty) \rightarrow[0, \infty)$ which are defined as

$$
\phi(t)=2 t \quad \text { and } \quad \psi(t)=\frac{1}{2} t(t \in[0, \infty))
$$

Clearly, $\phi \in \Phi$ and $\psi \in \Psi$. Let $((1,0),(1,0))$ in $\mathcal{X}_{\leq}$

$$
p(\mathcal{S}(1,0), \mathcal{S}(1,0))=p((1,0),(1,0))=\max \{d(g(1,0),(1,0)), d(g(1,0), g(1,0))\}=0,
$$

and for $((0,1),(0,1)) \in \mathcal{X}_{\leq}$, we have

$$
p(\mathcal{S}(0,1), \mathcal{S}(0,1))=p((1,0),(1,0))=0
$$

and

$$
p((0,1),(0,1))=\max \{d(g(0,1),(0,1)), d(g(0,1), g(0,1))\}=2 .
$$

Therefore, for every $\left(\left(x_{1}, x_{2}\right),\left(y_{1}, y_{2}\right)\right) \in \mathcal{X}_{\leq}$, we have

$$
\phi p\left(\mathcal{S}\left(x_{1}, x_{2}\right), \mathcal{S}\left(y_{1}, y_{2}\right)\right) \leq \psi \phi\left(p\left(\left(x_{1}, x_{2}\right),\left(y_{1}, y_{2}\right)\right)\right) .
$$

Thus, all the conditions of Theorem 1 are satisfied implying thereby the existence of a fixed point of $\mathcal{S}$ which is indeed $(x, y)=(1,0)$. 
Theorem 2 Let $(\mathcal{X}, d, \preceq)$ be a complete partially ordered metric space equipped with a $w$-distance $p$ and $\mathcal{S}: \mathcal{X} \rightarrow \mathcal{X}$ be a nondecreasing mapping. Suppose that

(a) there exists $x_{0} \in \mathcal{X}$ such that $\left(x_{0}, \mathcal{S} x_{0}\right) \in \mathcal{X}_{\leq}$,

(b) there exist $\psi \in \Psi$ and $\phi \in \Phi$ such that

$$
\phi(p(\mathcal{S} x, \mathcal{S} y)) \leq \psi \phi(p(x, y))
$$

for all $(x, y) \in \mathcal{X}_{\leq}$, and

$\left(\mathrm{c}^{\prime \prime}\right)$ for every $y \in \mathcal{X}$ with $y \neq \mathcal{S} y$,

$$
\inf \{p(x, y)+p(x, \mathcal{S} x): x \in \mathcal{X}\}>0
$$

Then $\mathcal{F}_{\mathcal{S}} \neq \emptyset$. Moreover, if $x=\mathcal{S} x$, then $p(x, x)=0$.

Proof Observe that the sequence $\left\{\mathcal{S}^{n} x_{0}\right\}$ is a Cauchy sequence (in view of the proof of Theorem 1), and so there exists a point $x^{*}$ in $\mathcal{X}$ such that $\lim _{n \rightarrow \infty} \mathcal{S}^{n} x_{0}=x^{n}$. Since $\lim _{m, n \rightarrow \infty} d\left(\mathcal{S}^{n} x_{0}, \mathcal{S}^{m} x_{0}\right)=0$, therefore for each $\epsilon>0$, there exists $N_{\epsilon} \in \mathbb{N}$ such that $n>N_{\epsilon}$ implies $p\left(\mathcal{S}^{N_{\epsilon}} x_{0}, \mathcal{S}^{n} x_{0}\right)<\epsilon$. Since $\lim _{n \rightarrow \infty} \mathcal{S}^{n} x_{0}=x^{*}$ and $p(x, \cdot)$ is lower semi-continuous, therefore

$$
p\left(\mathcal{S}^{N_{\epsilon}} x_{0}, x^{*}\right) \leq \liminf _{n} p\left(\mathcal{S}^{N_{\epsilon}} x_{0}, \mathcal{S}^{n} x_{0}\right) \leq \epsilon
$$

Therefore, $p\left(\mathcal{S}^{N_{\epsilon}}, x^{*}\right) \leq \epsilon$. Set $\epsilon=\frac{1}{k}, N_{\epsilon}=n_{k}$ so that

$$
\lim _{k \rightarrow \infty} p\left(\mathcal{S}^{n_{k}} x_{0}, x^{\prime \prime}\right)=0
$$

Now, assume that $x^{\prime \prime} \neq \mathcal{S} x^{\prime \prime}$. Then due to hypothesis $\left(\mathrm{c}^{\prime \prime}\right)$, we have

$$
0<\inf \left\{p\left(x, x^{*}\right)+p(x, \mathcal{S} x): x \in \mathcal{X}\right\} \leq \inf \left\{p\left(\mathcal{S}^{n_{k}} x_{0}, x^{*}\right)+p\left(\mathcal{S}^{n_{k}} x_{0}, \mathcal{S}^{n_{k}+1} x_{0}\right): n \in \mathbb{N}\right\} \rightarrow 0
$$

as $n \rightarrow \infty$. This is a contradiction. Hence $x^{*}=\mathcal{S} x^{*}$.

If $\mathcal{S} x=x$, we have

$$
\phi p(x, x)=\phi(p(\mathcal{S} x, \mathcal{S} x)) \leq \psi \phi(p(x, x))<\phi p(x, x),
$$

which is a contradiction implying thereby $\phi p(x, x)=0$ so that $p(x, x)=0$. This completes the proof.

Corollary 5 Replacing condition ( $\mathrm{c}^{\prime \prime}$ ) by hypothesis (c) or ( $\mathrm{c}^{\prime}$ ) of Corollary 2 (also of Corollary 3 or Corollary 4) the fixed point of $\mathcal{S}$ continues to exist.

In what follows, we give a sufficient condition for the uniqueness of a fixed point in Theorem 1 which runs as follows:

(A) for every $x, y \in \mathcal{X}$, there exists a lower bound or an upper bound.

In [18], it is proved that condition (A) is equivalent to the following one:

(B) for every $x, y \in \mathcal{X}$, there exists $z=c(x, y) \in \mathcal{X}$ for which $(x, z) \in \mathcal{X}_{\leq}$and $(y, z) \in \mathcal{X}_{\leq}$. 
Theorem 3 With the addition of condition (B) to the hypotheses of Theorem 1 (or Theorem 2), the fixed point of $\mathcal{S}$ turns out to be unique. Moreover,

$$
\lim _{n \rightarrow \infty} \mathcal{S}^{n}(x)=x^{*}
$$

for every $x \in \mathcal{X}$ provided $x^{*} \in \mathcal{F}_{\mathcal{S}}$, i.e., the map $\mathcal{S}: \mathcal{X} \rightarrow \mathcal{X}$ is a Picard operator.

Proof Following the proof of Theorem 1, $\mathcal{F}_{\mathcal{S}} \neq \emptyset$. Suppose there exist two fixed points $x^{*}$ and $y^{*}$ of $\mathcal{S}$ in $\mathcal{X}$. We prove that

$$
p\left(y^{*}, x^{*}\right)=0
$$

We distinguish two cases.

Case 1: If $\left(y^{*}, x^{*}\right) \in \mathcal{X}_{\leq}$. Suppose that $p\left(y^{*}, x^{*}\right)>0$, then by using condition (b) and the property of $\psi$, we get

$$
\phi p\left(y^{*}, x^{*}\right)=\phi p\left(\mathcal{S} y^{*}, \mathcal{S} x^{*}\right) \leq \psi \phi\left(p\left(y^{*}, x^{*}\right)\right)<\phi p\left(y^{*}, x^{*}\right)
$$

which is a contradiction. Therefore we have (9).

Also, in view of Theorem 1, we have

$$
p\left(y^{*}, y^{*}\right)=0
$$

On using (9), (10) and Lemma 3, we have $y^{*}=x^{*}$, i.e., the fixed point of $\mathcal{S}$ is unique.

Case 2: If $\left(x^{*}, y^{*}\right) \notin \mathcal{X}_{\leq}$, then owing to condition (B), there exists $z \in \mathcal{X}$ such that $\left(x^{*}, z\right) \in$ $\mathcal{X}_{\leq}$and $\left(y^{\prime \prime}, z\right) \in \mathcal{X}_{\leq}$. As $\left(z, x^{\prime \prime}\right) \in \mathcal{X}_{\leq}$and $\left(y^{\prime \prime}, z\right) \in \mathcal{X}_{\leq}$, proceeding along the lines of the proof of Theorem 1, we can prove

$$
\lim _{n \rightarrow \infty} p\left(\mathcal{S}^{n} z, x^{*}\right)=0 \quad \text { and } \quad \lim _{n \rightarrow \infty} p\left(\mathcal{S}^{n} z, y^{*}\right)=0 .
$$

By using Lemma 3, we infer that $y^{*}=x^{*}$, i.e., the fixed point of $\mathcal{S}$ is unique. Now, we prove

$$
\lim _{n \rightarrow \infty} \mathcal{S}^{n}(x)=x^{*}
$$

for every $x \in \mathcal{X}$ provided $x^{*} \in \mathcal{F}_{\mathcal{S}}$.

Let $x \in \mathcal{X}$ and $\left(x_{0}, x\right) \in \mathcal{X}_{\leq}$. Proceeding along the lines of the proof of Theorem 1, we can prove $\lim _{n \rightarrow \infty} p\left(\mathcal{S}^{n} x_{0}, \mathcal{S}^{n} x\right)=0$, and owing to $x^{n} \in \mathcal{F}_{\mathcal{S}}$ and $p$ is a $w$-distance (lower semi-continuous), then $\lim _{n \rightarrow \infty} p\left(\mathcal{S}^{n} x_{0}, x^{*}\right)=0$, by Lemma 3 , we get

$$
\lim _{n \rightarrow \infty} \mathcal{S}^{n} x=x^{*}
$$

Suppose $x \in \mathcal{X}$ and $\left(x_{0}, x\right) \notin \mathcal{X}_{\leq}$. Owing to condition (B), there exists some $z$ in $\mathcal{X}$ such that $\left(x_{0}, z\right) \in \mathcal{X}_{\leq}$and $(x, z) \in \mathcal{X}_{\leq}$.

Since $\left(x_{0}, z\right) \in \mathcal{X}_{\leq}$and $(x, z) \in \mathcal{X}_{\leq}$, by using condition (b) (proceeding along the lines of the proof of Theorem 1), we can prove $\lim _{n \rightarrow \infty} p\left(\mathcal{S}^{n} x_{0}, \mathcal{S}^{n} z\right)=0$ and $\lim _{n \rightarrow \infty} p\left(\mathcal{S}^{n} z\right.$, $\left.\mathcal{S}^{n} x\right)=0$. 
By the triangular inequality, we can write

$$
p\left(\mathcal{S}^{n} x_{0}, \mathcal{S}^{n} x\right) \leq p\left(\mathcal{S}^{n} x_{0}, \mathcal{S}^{n} z\right)+p\left(\mathcal{S}^{n} z, \mathcal{S}^{n} x\right)
$$

Letting $n \rightarrow \infty$, we get $\lim _{n \rightarrow \infty} p\left(\mathcal{S}^{n} x_{0}, \mathcal{S}^{n} x\right)=0$, and also $p$ is a $w$-distance (lower semicontinuous), we have $\lim _{n \rightarrow \infty} p\left(\mathcal{S}^{n} x_{0}, x^{*}\right)=0$, which due to Lemma 3 implies

$$
\lim _{n \rightarrow \infty} \mathcal{S}^{n} x=x^{*}
$$

This completes the proof.

The following example demonstrates Theorem 3.

Example 7 Let $\mathcal{X}=\{0\} \cup\left\{\frac{1}{2^{n}}: n \geq 1\right\}$, which $(\mathcal{X}, d, \preceq)$ is a complete partially ordered metric space with the usual metric $d$ and the usual order ' $\preceq$.' Clearly, condition (B) holds in $\mathcal{X}$. We define $p: \mathcal{X} \times \mathcal{X} \rightarrow[0, \infty)$ by $p(x, y)=y$. Let $\phi$ and $\psi$ be the mappings with $\phi, \psi:[0, \infty) \rightarrow[0, \infty)$ defined by

$$
\phi(t)= \begin{cases}2, & \text { if } t>1 \\ 2 t, & \text { if } t \in[0,1]\end{cases}
$$

and $\psi(t)=\frac{1}{2} t$. Obviously, $\phi \in \Phi$ and $\psi \in \Psi$. Assume that $\mathcal{S}: \mathcal{X} \rightarrow \mathcal{X}$ by $\mathcal{S} x=\frac{x}{8}$ for any $x \in \mathcal{X}$. It is easy to see that $\mathcal{S}$ is nondecreasing. Also, there is $x_{0}=0$ in $\mathcal{X}$ such that $\left(x_{0}, \mathcal{S} x_{0}\right) \in \mathcal{X}_{\leq}$, and $\mathcal{S}$ satisfies $\left(\mathrm{c}^{\prime}\right)$. Also, for any $n \in \mathbb{N}$, we have $\frac{1}{2^{n}} \neq \mathcal{S}\left(\frac{1}{2^{n}}\right)$. So, for arbitrary $n \in \mathbb{N}$, we have

$$
\inf \left\{p\left(\frac{1}{2^{m}}, \frac{1}{2^{n}}\right)+p\left(\frac{1}{2^{m}}, \frac{1}{2^{m+1}}\right): m \in \mathbb{N}\right\}=\frac{1}{2^{n}}>0 .
$$

Now, we show that $\mathcal{S}$ satisfies (b).

$$
\begin{aligned}
\phi p(\mathcal{S} x, \mathcal{S} y) & =\phi\left(\frac{1}{8} y\right) \\
& =2\left(\frac{y}{8}\right) \\
& =\frac{y}{4} \quad(\text { since } y<1, y \in \mathcal{X}) \\
& \leq y=\psi(2 y)=\psi(\phi(y))=\psi(\phi(p(x, y)) .
\end{aligned}
$$

Thus, all the conditions of Theorem 3 are satisfied and $x=0$ is the unique fixed point for $\mathcal{S}$. Moreover, $\lim _{n \rightarrow \infty} \mathcal{S}^{n}(x)=\lim _{n \rightarrow \infty} \frac{x}{8^{n}}=0$.

Corollary 6 With the addition of condition (B) to the hypotheses of Corollary 1 (or Corollaries $2,3,4,5)$ the fixed point of $\mathcal{S}$ turns out to be unique. Moreover,

$$
\lim _{n \rightarrow \infty} \mathcal{S}^{n}(x)=x^{*}
$$

for every $x \in \mathcal{X}$ provided $x^{*} \in \mathcal{F}_{\mathcal{S}}$, i.e., the map $\mathcal{S}: \mathcal{X} \rightarrow \mathcal{X}$ is a Picard operator. 
Our next example highlights the role of condition $\left(\mathrm{c}^{\prime \prime}\right)$ in Theorem 2.

Example 8 Consider $\mathcal{X}=[0,1]$, which is a complete metric space with the usual metric $d(x, y)=|x-y|$ for all $x, y \in \mathcal{X}$. Moreover, by defining $p(x, y)=2|x-y|, p$ is a $w$-distance on $(\mathcal{X}, d)$. We consider $\mathcal{X}_{\leq}$as follows:

$$
\mathcal{X}_{\leq}=\left\{(x, y) \in \mathcal{X} \times \mathcal{X}: x=y \text { or } x, y \in\left\{\frac{1}{2^{n}}: n=1,2,3, \ldots\right\}\right\}
$$

where $\preceq$ is the usual ordering.

Let $\mathcal{S}: \mathcal{X} \rightarrow \mathcal{X}$ be given by

$$
\mathcal{S}(x)= \begin{cases}\frac{1}{2^{n+1}}, & \text { if } x=\frac{1}{2^{n}} \\ \frac{1}{2}, & \text { otherwise }\end{cases}
$$

Obviously, $\mathcal{S}$ is a nondecreasing map. Also, there is $x_{0}=\frac{1}{2}$ in $\mathcal{X}$ such that $\left(x_{0}, \mathcal{S} x_{0}\right) \in \mathcal{X}_{\leq}$.

We now show that $\mathcal{S}$ satisfies (b) with $\phi, \psi:[0, \infty) \rightarrow[0, \infty)$ which are defined as

$$
\phi(t)=\left\{\begin{array}{ll}
1, & \text { if } t>1, \\
t^{\frac{1}{3}}, & \text { if } t \in[0,1],
\end{array} \quad \text { and } \quad \psi(t)=\frac{1}{2^{\frac{1}{3}}} t \quad(t \in[0, \infty))\right.
$$

Clearly, $\phi \in \Phi$ and $\psi \in \Psi$. If $x=y$, condition (b) is satisfied.

Now, let $x=\frac{1}{2^{n}}$ and $y=\frac{1}{2^{m}}(m>n)$. Then we have

$$
\begin{aligned}
\phi p(\mathcal{S} x, \mathcal{S} y) & =\phi\left(\frac{2\left(2^{m}-2^{n}\right)}{2^{m+n+1}}\right) \\
& =\phi\left(\frac{1-\frac{2^{n}}{2^{m}}}{2^{n}}\right) \\
& =\left(\frac{1-\frac{2^{n}}{2^{m}}}{2^{n}}\right)^{\frac{1}{3}} \\
& =\psi \phi(p(x, y))
\end{aligned}
$$

so that condition (b) is satisfied.

Since $0 \neq \mathcal{S} 0$, we have

$$
\inf \left\{p\left(\frac{1}{2^{n}}, 0\right)+p\left(\frac{1}{2^{n}}, \frac{1}{2^{n+1}}\right): n \in \mathbb{N}\right\}=\inf \left\{\frac{2}{2^{n}}+\frac{2}{2^{n+1}}: n \in \mathbb{N}\right\}=0
$$

Thus, all the conditions of Theorem 2 are satisfied except $\left(\mathrm{c}^{\prime \prime}\right)$.

Clearly, $\mathcal{S}$ has got no fixed point in $\mathcal{X}$.

\section{$3(\gamma, \psi, p)$-contractive maps}

In this section we state some results in a partial ordered metric space with $(\gamma, \psi, p)$ contractive maps. In Section 2 we considered the condition nondecreasing for the function $\mathcal{S}$, but in this section we will prove some theorems by replacing the condition nondecreasing to monotonicity for the function. 
Theorem 4 Let $(\mathcal{X}, d, \preceq)$ be a complete partially ordered metric space equipped with a $w$-distance $p$ and $\mathcal{S}: \mathcal{X} \rightarrow \mathcal{X}$ be a monotone mapping. Suppose that

(a) there exists $x_{0} \in \mathcal{X}$ such that $\left(x_{0}, \mathcal{S} x_{0}\right) \in \mathcal{X}_{\leq}$,

(b) there exist $\psi \in \Psi$ and $\gamma \in \Gamma$ such that

$$
\gamma\left(p\left(\mathcal{S} x, \mathcal{S}^{2} x\right)\right) \leq \psi \gamma(p(x, \mathcal{S} x))
$$

for all $(x, \mathcal{S} x) \in \mathcal{X}_{\leq}$,

(c) either $\mathcal{S}$ is orbitally continuous at $x_{0}$ or

$\left(c^{\prime}\right) \mathcal{S}$ is orbitally $\mathcal{X}_{\leq}$-continuous and there exists a subsequence $\left\{\mathcal{S}^{n_{k}} x_{0}\right\}$ of $\left\{\mathcal{S}^{n} x_{0}\right\}$ converging to $x^{*}$ such that $\left(\mathcal{S}^{n_{k}} x_{0}, x^{*}\right) \in \mathcal{X}_{\leq}$for any $k \in N$.

Then $\mathcal{F}_{\mathcal{S}} \neq \emptyset$. Moreover, if $x=\mathcal{S} x$, then $p(x, x)=0$.

Proof If $x_{0}=\mathcal{S} x_{0}$ for some $x_{0} \in \mathcal{X}$, then there is nothing to prove. Otherwise, let there be $x_{0} \in \mathcal{X}$ such that $x_{0} \neq \mathcal{S} x_{0}$, and $\left(x_{0}, \mathcal{S} x_{0}\right) \in \mathcal{X}_{\leq}$. Owing to monotonicity of $\mathcal{S}$, we can write $\left(\mathcal{S} x_{0}, \mathcal{S}^{2} x_{0}\right) \in \mathcal{X}_{\leq}$. Continuing this process inductively, we obtain

$$
\left(\mathcal{S}^{n} x_{0}, \mathcal{S}^{n+1} x_{0}\right) \in \mathcal{X}_{\leq}
$$

for any $n, m \in N$. Now, we proceed to show that

$$
\lim _{n \rightarrow \infty} p\left(\mathcal{S}^{n} x_{0}, \mathcal{S}^{n+1} x_{0}\right)=0 .
$$

On using condition (b) and the properties of $\gamma, \psi$, we get

$$
\begin{aligned}
\gamma\left(p\left(\mathcal{S}^{n} x_{0}, \mathcal{S}^{n+1} x_{0}\right)\right) & \leq \psi \gamma\left(p\left(\mathcal{S}^{n-1} x_{0}, \mathcal{S}^{n} x_{0}\right)\right) \\
& \leq \psi^{2} \gamma\left(p\left(\mathcal{S}^{n-2} x_{0}, \mathcal{S}^{n-1} x_{0}\right)\right) \\
& \leq \cdots \\
& \leq \psi^{n-m} \gamma\left(p\left(\mathcal{S}^{m} x_{0}, \mathcal{S}^{m+1} x_{0}\right)\right) \\
& \leq \cdots \\
& \leq \psi^{n} \gamma\left(p\left(x_{0}, \mathcal{S} x_{0}\right)\right) .
\end{aligned}
$$

By using Lemma 1, we have $\lim _{n \rightarrow \infty} \gamma p\left(\mathcal{S}^{n} x_{0}, \mathcal{S}^{n+1} x_{0}\right)=0$ so that by Lemma 2 , we have

$$
\lim _{n \rightarrow \infty} p\left(\mathcal{S}^{n} x_{0}, \mathcal{S}^{n+1} x_{0}\right)=0,
$$

which establishes (11).

Similarly, we can show

$$
\lim _{n \rightarrow \infty} p\left(\mathcal{S}^{n+1} x_{0}, \mathcal{S}^{n} x_{0}\right)=0 .
$$

Now, we proceed to show that $\left\{\mathcal{S}^{n} x_{0}\right\}$ is a Cauchy sequence. By the triangle inequality, the continuity of $\gamma$ and (11), we have

$$
\gamma p\left(\mathcal{S}^{n} x_{0}, \mathcal{S}^{n+2} x_{0}\right) \leq \gamma\left(p\left(\mathcal{S}^{n} x_{0}, \mathcal{S}^{n+1} x_{0}\right)+p\left(\mathcal{S}^{n+1} x_{0}, \mathcal{S}^{n+2} x_{0}\right)\right) \rightarrow 0
$$


as $n \rightarrow \infty$ so that $\lim _{n \rightarrow \infty} \gamma p\left(\mathcal{S}^{n} x_{0}, \mathcal{S}^{n+2} x_{0}\right)=0$, which amounts to say that

$$
\lim _{n \rightarrow \infty} p\left(\mathcal{S}^{n} x_{0}, \mathcal{S}^{n+2} x_{0}\right)=0
$$

By induction, for any $k>0$, we have

$$
\lim _{n \rightarrow \infty} p\left(\mathcal{S}^{n} x_{0}, \mathcal{S}^{n+k} x_{0}\right)=0
$$

So, by Lemma $3,\left\{\mathcal{S}^{n} x_{0}\right\}$ is a Cauchy sequence and due to the completeness of $\mathcal{X}$, there exists $x^{*} \in \mathcal{X}$ such that $\lim _{n \rightarrow \infty} \mathcal{S}^{n} x_{0}=x^{*}$.

If (c) or ( $\left.c^{\prime}\right)$ holds, then proceeding along the lines of the proof of Theorem 1, we can show that

$$
\mathcal{S} x^{*}=x^{*}
$$

If $\mathcal{S} x=x$, we have

$$
\gamma p(x, x)=\gamma\left(p\left(\mathcal{S} x, \mathcal{S}^{2} x\right)\right) \leq \psi \gamma(p(x, \mathcal{S} x))<\gamma p(x, x)
$$

which is a contradiction so that $\gamma p(x, x)=0$, implying thereby $p(x, x)=0$. This completes the proof.

Theorem 5 Let $(\mathcal{X}, d, \preceq)$ be a complete partially ordered metric space equipped with a $w$-distance $p$ and $\mathcal{S}: \mathcal{X} \rightarrow \mathcal{X}$ be a monotone mapping. Suppose that

(a) there exists $x_{0} \in \mathcal{X}$ such that $\left(x_{0}, \mathcal{S} x_{0}\right) \in \mathcal{X}_{\leq}$,

(b) there exist $\psi \in \Psi$ and $\gamma \in \Gamma$ such that

$$
\gamma\left(p\left(\mathcal{S} x, \mathcal{S}^{2} x\right)\right) \leq \psi \gamma(p(x, \mathcal{S} x))
$$

for all $(x, \mathcal{S} x) \in \mathcal{X}_{\leq}$, and

$\left(c^{\prime \prime}\right)$ for every $y \in \mathcal{X}$ with $y \neq \mathcal{S} y$,

$$
\inf \{p(x, y)+p(x, \mathcal{S} x): x \in \mathcal{X}\}>0
$$

Then $\mathcal{F}_{\mathcal{S}} \neq \emptyset$. Moreover, if $x=\mathcal{S} x$, then $p(x, x)=0$.

Proof Proceeding along the lines of the proof of Theorem 4, the sequence $\left\{\mathcal{S}^{n} x_{0}\right\}$ is a Cauchy sequence, and so there exists a point $x^{*}$ in $\mathcal{X}$ such that $\lim _{n \rightarrow \infty} \mathcal{S}^{n} x_{0}=x^{*}$. Since $\lim _{m, n \rightarrow \infty} d\left(\mathcal{S}^{n} x_{0}, \mathcal{S}^{m} x_{0}\right)=0$, therefore for each $\epsilon>0$, there exists $N_{\epsilon} \in \mathbb{N}$ such that $n>$ $N_{\epsilon}$ implies $p\left(\mathcal{S}^{N_{\epsilon}} x_{0}, \mathcal{S}^{n} x_{0}\right)<\epsilon$. As $\lim _{n \rightarrow \infty} \mathcal{S}^{n} x_{0}=x^{*}$ and $p(x, \cdot)$ is lower semi-continuous, therefore

$$
p\left(\mathcal{S}^{N_{\epsilon}} x_{0}, x^{*}\right) \leq \liminf _{n} p\left(\mathcal{S}^{N_{\epsilon}} x_{0}, \mathcal{S}^{n} x_{0}\right) \leq \epsilon
$$

Therefore $p\left(\mathcal{S}^{N_{\epsilon}} x_{0}, x^{*}\right) \leq \epsilon$. Setting $\epsilon=\frac{1}{k}, N_{\epsilon}=n_{k}$, we have

$$
\lim _{k \rightarrow \infty} p\left(\mathcal{S}^{n_{k}} x_{0}, x^{*}\right)=0
$$


Now, assume that $x^{* \prime} \neq \mathcal{S} x^{*}$. Then by hypothesis $\left(\mathrm{c}^{\prime \prime}\right)$, we have

$$
0<\inf \left\{p\left(x, x^{\prime \prime}\right)+p(x, \mathcal{S} x): x \in \mathcal{X}\right\} \leq \inf \left\{p\left(\mathcal{S}^{n} x_{0}, x^{*}\right)+p\left(\mathcal{S}^{n} x_{0}, \mathcal{S}^{n+1} x_{0}\right): n \in \mathbb{N}\right\} \rightarrow 0
$$

as $n \rightarrow \infty$. This is a contradiction so that $x^{*}=\mathcal{S} x^{*}$.

If $\mathcal{S} x=x$, we have

$$
\gamma p(x, x)=\gamma\left(p\left(\mathcal{S} x, \mathcal{S}^{2} x\right)\right) \leq \psi \gamma(p(x, \mathcal{S} x))<\gamma p(x, x)
$$

which is a contradiction so that $\gamma p(x, x)=0$, yielding thereby $p(x, x)=0$. This completes the proof.

Our next example demonstrates Theorem 5 which exhibits the utility of this theorem over Theorems 1 and 2 .

Example 9 Consider $\mathcal{X}=[0,1]$, which is a complete metric space with the usual metric $d(x, y)=|x-y|$ for all $x, y \in \mathcal{X}$. Moreover, by defining $p(x, y)=y, p$ is a $w$-distance on $(\mathcal{X}, d)$. We consider $\mathcal{X}_{\leq}$as follows:

$$
\mathcal{X}_{\leq}=\left\{(x, y) \in \mathcal{X} \times \mathcal{X}: x=y \text { or } x, y \in\{0\} \cup\left\{\frac{1}{n}: n=1,2,3, \ldots\right\}\right\},
$$

where $\preceq$ is the usual ordering.

Let $\mathcal{S}: \mathcal{X} \rightarrow \mathcal{X}$ be given by

$$
\mathcal{S}(x)= \begin{cases}\frac{1}{n+1}, & \text { if } x=\frac{1}{n} \\ 0, & \text { if } x=0 \\ 1, & \text { otherwise. }\end{cases}
$$

Obviously, $\mathcal{S}$ is a monotone map. Also, there is $x_{0}=0$ in $\mathcal{X}$ such that $0=x_{0} \preceq \mathcal{S} x_{0}=0$, i.e., $\left(x_{0}, \mathcal{S} x_{0}\right) \in \mathcal{X}_{\leq}$.

We now show that $\mathcal{S}$ satisfies (b) with $\psi, \gamma:[0, \infty) \rightarrow[0, \infty)$ which are respectively defined as $\psi(t)=\frac{1}{3} t$ and

$$
\gamma(t)= \begin{cases}t^{\frac{1}{t}}, & \text { if } t>0 \\ 0, & \text { if } t=0 .\end{cases}
$$

Clearly, $\psi \in \Psi$ and $\gamma \in \Gamma$. If $x=0$ as $(0, \mathcal{S} 0) \in \mathcal{X}_{\leq}$, it is easy to show that condition (b) is satisfied.

Suppose $x=\frac{1}{n}$ as $\left(\frac{1}{n}, \mathcal{S}\left(\frac{1}{n}\right)\right) \in \mathcal{X}_{\leq}$, one gets

$$
\begin{aligned}
\gamma p\left(\mathcal{S} x, \mathcal{S}^{2} x\right) & =\gamma\left(\frac{1}{n+2}\right)=\left(\frac{1}{n+2}\right)^{\frac{1}{n+2}} \leq \frac{1}{3}\left(\frac{1}{n+1}\right)^{\frac{1}{n+1}} \\
& =\frac{1}{3} \gamma\left(p\left(\frac{1}{n}, \mathcal{S}\left(\frac{1}{n}\right)\right)\right)=\psi \gamma(p(x, \mathcal{S} x))
\end{aligned}
$$

as for any $n \in \mathbb{N}$, we have $\left(\frac{n+1}{n+2}\right)^{n+1} \frac{1}{n+2} \leq \frac{1}{3}$. Hence condition (b) is satisfied. 
If $y>0$, we have $y \neq \mathcal{S} y$ so that

$$
\inf \{p(x, y)+p(x, \mathcal{S} x): x \in \mathcal{X}\}=\inf \{y+\mathcal{S} x: x \in \mathcal{X}\}>0 .
$$

Thus, all the conditions of Theorem 5 are satisfied and $x=0$ is a fixed point of $\mathcal{S}$.

But if $x=y \neq 0, \frac{1}{n}$ is chosen, then $\gamma p(\mathcal{S} x, \mathcal{S} y)=1$ and $\psi \gamma(p(x, y))=\frac{1}{3} y^{\frac{1}{y}}$ so that $\gamma p(\mathcal{S} x, \mathcal{S} y)>\psi \gamma(p(x, y))$. Hence, condition (b) of Theorems 1 and 2 does not hold.

Theorem 6 Let $(\mathcal{X}, d, \preceq)$ be a complete partially ordered metric space equipped with a $w$-distance $p$ and $\mathcal{S}: \mathcal{X} \rightarrow \mathcal{X}$ be a monotone mapping. Suppose that

(a) there exists $x_{0} \in \mathcal{X}$ such that $\left(x_{0}, \mathcal{S} x_{0}\right) \in \mathcal{X}_{\leq}$,

$\left(b_{1}\right)$ there exist $\gamma \in \Gamma$ and $k \in\left[0, \frac{1}{2}\right)$ such that

$$
\gamma(p(\mathcal{S} x, \mathcal{S} y)) \leq k\{\gamma p(x, \mathcal{S} x)+\gamma p(y, \mathcal{S} y)\}
$$

for all $(x, y) \in \mathcal{X}_{\leq}$

$\left(\mathrm{c}^{\prime \prime}\right)$ for every $y \in \mathcal{X}$ with $y \neq \mathcal{S} y$,

$$
\inf \{p(x, y)+p(x, \mathcal{S} x): x \in \mathcal{X}\}>0
$$

Then $\mathcal{F}_{\mathcal{S}} \neq \emptyset$. Moreover, if $x=\mathcal{S} x$, then $p(x, x)=0$.

Proof For $x \in \mathcal{X}$, set $y=\mathcal{S} x$ and $\alpha=\frac{k}{1-k}$. Then we have $(x, \mathcal{S} x) \in \mathcal{X}_{\leq}$and $\alpha \in[0,1)$. On using condition $\left(\mathrm{b}_{1}\right)$, we get

$$
\gamma\left(p\left(\mathcal{S} x, \mathcal{S}^{2} x\right)\right) \leq k\left\{\gamma p(x, \mathcal{S} x)+\gamma p\left(\mathcal{S} x, \mathcal{S}^{2} x\right)\right\}
$$

or

$$
\gamma\left(p\left(\mathcal{S} x, \mathcal{S}^{2} x\right)\right) \leq \alpha \gamma p(x, \mathcal{S} x)
$$

Therefore, by choosing $\psi(t)=\alpha t$, all the conditions of Theorem 5 are satisfied ensuring the conclusions of the theorem.

The set of all subadditive functions $\gamma$ in $\Gamma$ is denoted by $\Gamma^{\prime}$.

Theorem 7 Let $(\mathcal{X}, d, \preceq)$ be a complete partially ordered metric space equipped with a $w$-distance $p$ and $\mathcal{S}: \mathcal{X} \rightarrow \mathcal{X}$ be a monotone mapping. Suppose that

(a) there exists $x_{0} \in \mathcal{X}$ such that $\left(x_{0}, \mathcal{S} x_{0}\right) \in \mathcal{X}_{\leq}$,

$\left(\mathrm{b}_{2}\right)$ there exist $\gamma \in \Gamma^{\prime}$ and $k \in\left[0, \frac{1}{2}\right)$ such that

$$
\gamma\left(p\left(\mathcal{S} x, \mathcal{S}^{2} x\right)\right) \leq k \gamma\left(p\left(x, \mathcal{S}^{2} x\right)\right)
$$

for all $(x, \mathcal{S} x) \in \mathcal{X}_{\leq}$, and

$$
\inf \{p(x, y)+p(x, \mathcal{S} x): x \in \mathcal{X}\}>0
$$

for every $y \in \mathcal{X}$ with $y \neq \mathcal{S} y$. Then $\mathcal{F}_{\mathcal{S}} \neq \emptyset$. Moreover, if $x=\mathcal{S} x$, then $p(x, x)=0$. 
Proof Set $\alpha=\frac{k}{1-k}$, then $\alpha \in[0,1)$. On using condition $\left(\mathrm{b}_{2}\right)\left(\right.$ as $\left.\gamma \in \Gamma^{\prime}\right)$, we have

$$
\begin{aligned}
\gamma\left(p\left(\mathcal{S} x, \mathcal{S}^{2} x\right)\right) & \leq k \gamma\left(p\left(x, \mathcal{S}^{2} x\right)\right) \leq k \gamma\left(p(x, \mathcal{S} x)+p\left(\mathcal{S} x, \mathcal{S}^{2} x\right)\right) \\
& \leq k \gamma p(x, \mathcal{S} x)+k \gamma p\left(\mathcal{S} x, \mathcal{S}^{2} x\right)
\end{aligned}
$$

Thus, $\gamma\left(p\left(\mathcal{S} x, \mathcal{S}^{2} x\right)\right) \leq \alpha \gamma(p(x, \mathcal{S} x))$.

Therefore, by choosing $\psi(t)=\alpha t$, all the conditions of Theorem 5 are satisfied ensuring the conclusions of the theorem.

Our final example demonstrates Theorem 4.

Example 10 Consider $\mathcal{X}=\{(1,0,0),(0,1,0),(0,0,1)\}$, which is a complete metric space with $d\left(\left(x_{1}, x_{2}, x_{3}\right),\left(y_{1}, y_{2}, y_{3}\right)\right)=\left|x_{1}-y_{1}\right|+\left|x_{2}-y_{2}\right|+\left|x_{3}-y_{3}\right|$. Define $g: \mathcal{X} \rightarrow \mathcal{X}$ by $g(1,0,0)=$ $(1,0,0)$ and $g(0,1,0)=(0,0,1)$, and $g(0,0,1)=(0,0,1)$, which is a continuous map. Moreover, by defining

$$
p\left(\left(x_{1}, x_{2}, x_{3}\right),\left(y_{1}, y_{2}, y_{3}\right)\right)=\max \left\{d\left(g\left(x_{1}, x_{2}, x_{3}\right),\left(y_{1}, y_{2}, y_{3}\right)\right), d\left(g\left(x_{1}, x_{2}, x_{3}\right), g\left(y_{1}, y_{2}, y_{3}\right)\right)\right\}
$$

$p$ is a $w$-distance on $(\mathcal{X}, d)$. For $(x, y)$ and $(z, t)$ in $\mathcal{X}$, define ' $\preceq$ ' as follows: $\left(x_{1}, x_{2}, x_{3}\right) \preceq$ $\left(y_{1}, y_{2}, y_{3}\right) \Leftrightarrow x_{1} \leq y_{1}$ and $x_{2} \leq y_{2}$ and $x_{3} \leq y_{3}$, where ' $\leq$ ' is the usual ordering and

$$
\mathcal{X}_{\preceq}=\{((1,0,0),(1,0,0)),((0,1,0),(0,1,0)),((0,0,1),(0,0,1))\} .
$$

Define $\mathcal{S}: \mathcal{X} \rightarrow \mathcal{X}$ as

$$
\mathcal{S}(x, y)= \begin{cases}(1,0,0), & \text { if }(x, y)=(1,0,0) \\ (1,0,0), & \text { if }(x, y)=(0,1,0) \\ (0,0,1), & \text { if }(x, y)=(0,0,1)\end{cases}
$$

Clearly, $\mathcal{S}$ is a monotone and continuous map. Also, $(1,0,0) \preceq \mathcal{S}(1,0,0)=(1,0,0)$, i.e. $((1,0,0), \mathcal{S}(1,0,0)) \in \mathcal{X}_{\preceq}$.

We now show that $\mathcal{S}$ satisfies (b) with $\gamma, \psi:[0, \infty) \rightarrow[0, \infty)$ which are defined as

$$
\gamma(t)=t^{2} \quad \text { and } \quad \psi(t)=\frac{1}{4} t(t \in[0, \infty))
$$

Notice that $\gamma \in \Gamma$ and $\psi \in \Psi$. If $((1,0,0),(1,0,0))$ in $\mathcal{X}_{\leq}$, then

$$
\begin{aligned}
p\left(\mathcal{S}(1,0,0), \mathcal{S}^{2}(1,0,0)\right) & =p((1,0,0),(1,0,0)) \\
& =\max \{d(g(1,0,0),(1,0,0)), d(g(1,0,0), g(1,0,0))\}=0,
\end{aligned}
$$

and for $((0,0,1),(0,0,1)) \in \mathcal{X}_{\leq}$, we have

$$
\begin{aligned}
p\left(\mathcal{S}(0,0,1), \mathcal{S}^{2}(0,0,1)\right) & =p((0,0,1),(0,0,1)) \\
& =\max \{d(g(0,0,1),(0,0,1)), d(g(0,0,1), g(0,0,1))\}=0 .
\end{aligned}
$$


Therefore, for every $\left(\left(x_{1}, x_{2}, x_{3}\right), \mathcal{S}\left(x_{1}, x_{2}, x_{3}\right)\right) \in \mathcal{X}_{\leq}$, we have

$$
\gamma p\left(\mathcal{S}\left(x_{1}, x_{2}, x_{3}\right), \mathcal{S}^{2}\left(x_{1}, x_{2}, x_{3}\right)\right) \leq \psi \gamma\left(p\left(\left(x_{1}, x_{2}, x_{3}\right), \mathcal{S}\left(x_{1}, x_{2}, x_{3}\right)\right)\right)
$$

Thus, all the conditions of Theorem 4 are satisfied implying thereby the existence of fixed points of the map $\mathcal{S}$ which are indeed $(1,0,0)$ and $(0,0,1)$.

\section{Competing interests}

The authors declare that they have no competing interests.

\section{Authors' contributions}

All authors carried out the proof. All authors conceived of the study and participated in its design and coordination. All authors read and approved the final manuscript.

\section{Author details}

'Department of Mathematics, Aligarh Muslim University, Aligarh, 202002, India. ²Department of Applied Mathematics \& Humanities, S. V. National Institute of Technology, Surat, 395007, India.

\section{Acknowledgements}

The authors are thankful to the learned referees for their deep observations and pertinent suggestions, which greatly helped us to improve the paper significantly. The third author also thanks for the support of CSIR, Govt. of India, Grant No - 25(2882)/NS/12/EMR-II.

\section{Received: 18 October 2012 Accepted: 10 February 2013 Published: 1 March 2013}

\section{References}

1. Kada, O, Suzuki, T, Takahashi, W: Nonconvex minimization theorems and fixed point theorems in complete metric spaces. Math. Jpn. 44, 381-391 (1996)

2. Caristi, J: Fixed point theorems for mapping satisfying inwardness conditions. Trans. Am. Math. Soc. 215, 241-251 (1976)

3. Ekeland, I: Nonconvex minimization problem. Bull. Am. Math. Soc. 1, 443-474 (1979)

4. Takahashi, W: Existence theorems generalizing fixed point theorems for multivalued mappings. In: Thera, MA, Baillon, JB (eds.) Fixed Point Theory and Application. Pitman Research Notes in Mathematics Series, vol. 252, pp. 397-406. Wiley, New York (1991)

5. Ran, ACM, Reurings, MCB: A fixed point theorem in partially ordered sets and some applications to matrix equations. Proc. Am. Math. Soc. 132, 1435-1443 (2004)

6. Branciari, A: A fixed point theorem for mapping satisfying a general contractive condition of integral type. Int. J. Math. Math. Sci. 10, 531-536 (2002)

7. Vijayaraju, P, Rhoades, BE, Mohanraj, R: A fixed point theorem for pair of maps satisfying a general contractive condition of integral type. Int. J. Math. Math. Sci. 15, 2359-2364 (2005)

8. Kumar, S, Chugh, R, Kumar, R: Fixed point theorem for compatible mapping satisfying a contractive condition of integral type. Soochow J. Math. 33(2), 181-185 (2007)

9. Popa, V, Mocanu, M: A new view point in the study of fixed points for mappings satisfying a contractive condition of integral type. Bull. Inst. Politeh. Tasi, Ser. Mat. Mat. Teor. Fiz. 53(55), 269-280 (2007)

10. Suzuki, T: Meir-Keeler contractions of integral type are still Meir-Keeler contractions. Int. J. Math. Math. Sci. 2007, Article ID 39281 (2007)

11. Jachymski, J: Remarks on contractive conditions of integral type. Nonlinear Anal. 71, 1073-1081 (2009)

12. Razani, A, Mazlumi Nezhad, Z, Boujary, M: A fixed point theorem for w-distance. Appl. Sci. 11, 114-117 (2009)

13. Lakzian, H, Lin, I-J: The existence of fixed points for nonlinear contractive maps in metric spaces with $w$-distances. J. Appl. Math. 2012, Article ID 161470 (2012)

14. Hicks, TL, Rhoades, BE: A Banach type fixed-point theorem. Math. Jpn. 24(3), 327-330 (1979-1980)

15. Takahashi, W: Nonlinear Function Analysis. Yokohama Publishers, Yokohama (2000)

16. Khan, MS, Swaleh, M, Sessa, S: Fixed point theorems by altering distances between the points. Bull. Aust. Math. Soc 30, 1-9 (1984)

17. Suzuki, T: Several fixed point theorem in complete metric space. Yokohama Math. J. 44, 61-72 (1997)

18. Nieto, JJ, Rodríguez-López, R: Contractive mapping theorems in partially ordered sets and applications to ordinary differential equations. Order 22, 223-239 (2005) 